\title{
Cadmium im Schrott aus Müllverbrennungsanlagen
}

Zusammenfassung: Aufgrund des breiten Einsatzgebietes von Nickel-Cadmium (Ni-Cd) Batterien ist auch deren Gehalt in Siedlungsabfällen, die in Müllverbrennungsanlagen verbrannt werden, nicht vernachlässigbar. In dieser Arbeit wird die Konzentration an Cadmium im Eisenschrott, der aus Müllverbrennungsschlacke gewonnen wird, untersucht. Anhand von Verbrauchsdaten, Probenahmen auf MVAen und Laboranalysen werden folgende Ergebnisse erhalten: In einer Tonne Schrott findet man rund $0,8 \mathrm{~kg} \mathrm{Ni}-$ Cd-Akkumulatoren, die etwa $15 \%$ Cd enthalten. Daraus lässt sich eine durchschnittliche Konzentration von ca. 100 Gramm Cd pro Tonne Eisenschrott respektive ein Beitrag von 2-5 Gramm Cd je Tonne Restmüll berechnen. In Zukunft ist einerseits bei der Verwertung des Schrotts der relativ hohe Gehalt an Cd zu berücksichtigen; andererseits zeigen die Resultate, dass durchaus noch erhebliches Potential für die Verbesserung der separaten Sammlung von Ni-Cd Batterien besteht.

Cadmium in the scrap from waste incineration plants

Summary: The use of Ni-Cd batteries in consumer goods leads to a significant amount of these batteries in municipal solid waste for incineration. Cadmium contents of iron scrap produced from waste incineration bottom ask have been studied on the basis of consumption data, samples taken at waste incineration plants and laboratory analyses. This has yielded the following results: About 0.8 kilogram of Ni-Cd accumulators, containing about $15 \% \mathrm{Cd}$, is found in one tonne of scrap. Corresponding to an average content of about 100 grams of $\mathrm{Cd}$ per tonne of iron scrap, or 2-5 grams of $\mathrm{Cd}$ per tonne of residual waste. This relatively high Cd content has to be considered when utilising the scrap; the results also show that there is still a considerable potential for improving the separate collection of Ni-Cd batteries.

\section{Einleitung}

Bereits in den 80er Jahren wurde für einzelne Schwermetalle gezeigt, dass ein maßgeblicher Anteil ihres volkswirtschaftlichen Umsatzes schlussendlich über Siedlungsabfälle in Müllverbrennungsanlagen (MVA) gelangt und dort auf die Verbrennungsprodukte aufgeteilt wird (Brunner und Zobrist, 1983). Die Verteilung auf Schlacke, Rauchgasreinigungsprodukte, Abwasser und Reingas wird dabei durch drei Faktoren beeinflusst: 1. Die Verfahrenstechnik der Verbrennung und Rauchgasreinigung, 2. die chemisch-physikalischen Eigenschaften der Metalle und deren Speziierung, sowie 3. die Abfälle, in denen sich die Metalle befinden. Während die ersten beiden Faktoren einleuchten, sei kurz anhand eines Beispiels auf den dritten eingegangen: Ein massives Bleirohr wird die MVA in Form von Bleiklumpen über die Schlacke verlassen, während Blei, das zur Stabilisierung von PVC in einem Kunststoffartikel eingesetzt wurde, den Ofen mit dem Abgas verlässt und nach der Abgasreinigung zum größten Teil im Filterstaub endet.

Ein Hauptthema der Abfallwirtschaft der 80er Jahre waren Emissionen von Schwermetallen und Dioxinen aus MVAen. Diese Problematik wurde durch die Einführung sehr leistungsfähiger, aufwändiger Rauchgasreinigungssysteme gelöst. Damit wurden Schwermetalle und Dioxine jedoch nicht vernichtet, sondern nur in die Rückstände der Rauchgasreinigung transferiert. In der Folge wurden die festen Produkte der Verbrennung zu einem neuen Hauptproblem: Die an Schadstoffen reichen Stäube der Rauchgasreinigung mussten in Untertagedeponien entsorgt werden, die Schlacken auf Reststoffdeponien abgelagert werden.

In der vorliegenden Arbeit wird ein neues Schwermetallthema von MVAen untersucht, nämlich die Kontamination von Eisenschrott durch Cadmium: Die Verbrennungsschlacke wird üblicherweise nass aus dem Ofen abgezogen und über einen Magnetabscheider vom Eisen befreit. Dieser Schrott wird in Shredderbetrieben aufbereitet und in Stahlwerken verwertet. Erste Untersuchungen an MVAen haben gezeigt, dass infolge der relativ großen Menge an Ni-Cd-Akkumulatoren, die mit dem Restmüll in MVAen gelangen und nicht verbrennen, der Cadmiumgehalt des Eisenschrottes bei der Beurteilung der Umweltverträglichkeit nicht mehr vernachlässigt werden kann.

Ni-Cd-Batterien enthalten 11-22\% Cadmium (Kaufmann et al., 1997). Je nach Ladungszustand kommt ein Teil des Cadmiums - nie aber die gesamte Menge - in metallischer Form vor: die Akkus enthalten einen Cd-Überschuss um die $\mathrm{H}_{2}$-Bildung bei der Überladung zu verhindern. In den Zellen liegt das Cadmium in oxidierter Form als Cadmiumhydroxid $\mathrm{Cd}(\mathrm{OH})_{2}$ vor, welches oberhalb $300{ }^{\circ} \mathrm{C}$ in Cadmiumoxid $\mathrm{CdO}$ umgewandelt wird. Das Oxid sublimiert bei $1559{ }^{\circ} \mathrm{C}$. Als Elektrolyt ist Kalilauge in den Akkus enthalten. Organische Verbindungen befinden sich nicht in der Batteriefüllung. Eine Reduktion zu Cd0 oder die Bildung von Chloriden ist unter diesen Umständen nicht in großem Umfang zu erwarten. Es stellt sich daher die Frage, ob - ähnlich wie bei der MBA (Skutan und Brunner, 2009) - ein relevanter Teil des Cadmiums bei der Verbrennung in den Batterien bleibt und mit dem Magnetseparationsschrott ausgetragen wird. Die Antwort ist nicht nur wichtig für die Schrottverwertung, sondern auch für die Bilanzierung von MVAen, bei der die Verunreinigungen von Schrotten meis nicht berücksichtigt wird. Das Ziel der vorliegenden Arbeit war deshalb, Schrotte aus Müllverbrennungsanlagen auf den Gehalt an Batterien zu untersuchen, und die aufgefundenen Batterien auf Restcadmiumgehalte $\mathrm{zu}$ analysieren.

\section{Methoden}

Die Untersuchungen wurden an zwei österreichischen Müllverbrennungsanlagen durchgeführt. Die Versuche auf Anlage 1 (Versuch 1a und 1b) fanden im Juni 2002 statt (für Details siehe Vanzetta, 2002). Die Proben von Anlage 2 (Versuch 2) wurden im November 2007 während einer viertägigen Probenahmekampagne anlässlich der Erstellung einer Stoffbilanz gewonnen. 


\begin{tabular}{llllll}
\hline Tab. 1: Mengen von Input und Output bei der großtechnischen Aufbereitung der Schlacke für die Versuche 1a und 1b \\
\hline Versuch & $\begin{array}{l}\text { Input } \\
\text { Rostasche } \\
{[\mathbf{k g}]}\end{array}$ & $\begin{array}{l}\text { Output } \\
\text { Fe-Schrott }>\mathbf{5 0} \mathbf{~ m m} \\
{[\mathbf{k g}]}\end{array}$ & $\begin{array}{l}\text { Output } \\
\text { Fe-Schrott }<\mathbf{5 0 ~} \mathbf{~ m m} \\
{[\mathbf{k g}]}\end{array}$ & $\begin{array}{l}\text { Output } \\
\text { entschrottete Rest- } \\
\text { fraktion } \mathbf{5 0} \mathbf{~ m m} \\
{[\mathbf{k g}]}\end{array}$ & $\begin{array}{l}\text { Output } \\
\text { entschrottete Rest- } \\
\text { fraktion }<\mathbf{5 0} \mathbf{~ m m} \\
{[\mathbf{k g} ; \text { errechnet] }}\end{array}$ \\
\hline 1a & 24.300 & 770 & 320 & 1.900 & 21.310 \\
\hline 1b & 24.120 & 1.200 & 360 & 2.460 & 20.100 \\
\hline Summe 1a+b & 48.420 & 1.970 & 680 & 4.360 & 41.410 \\
\hline
\end{tabular}

\subsection{Abtrennung der Batterien aus den Schrotten}

Bei den Versuchen 1a und 1b wurden die Produkte einer Schlackenaufbereitungsanlage untersucht. Diese Anlage erzeugte zwei Siebfraktionen ( $>50 \mathrm{~mm}$ und $<50$ $\mathrm{mm}$ ). Aus jeder Siebfraktion wurde durch Magnetseparation Schrott abgeschieden. Aus beiden Schrotten wurden von Hand alle Batterien aussortiert. Die in den Versuchen aufbereiteten und sortierten Mengen an Schlache und Behandlungsprodukten sind in Tabelle 1 dargestellt.

Beim Versuch 2 wurde über 4 Tage der beim Magnetabscheider entstehende Schrott beprobt. Es wurden 40 Inkremente entnommen und zu vier Mischproben mit je ca. $152 \mathrm{~kg}$ vereint. Aus diesen Proben wurden die Batterien ebenfalls von Hand ausgelesen.

\subsection{Probenaufbereitung und Analyse}

Die Batterien wurden nach den Typen „Alkali-Mangan“, „Zink-Kohle“, „Nickel-Cadmium“ und "Nickel-Metallhydrid“ sortiert. Um sie zu identifizieren, mussten sie geöffnet werden. Bei den Versuchen auf Anlage 1 wurden die Batterien radial durchgeschnitten, was zu Verlusten an Batterieinhalt führte. Die Batterien von Anlage 2 wurden nur so weit notwendig angeschnitten. Die sichtbaren Erkennungsmerkmale der einzelnen Batterietypen sind: (1) die starken nahtlosen Becher mit im Kopf integriertem Überdruckventil bei Sekundärzellen sowie die häufig anzutreffenden Lötfahnen (zur Zusammenstellung von „Akku-Packs“), (2) die spiralig aufgerollten Sinterelektroden im Inneren der Sekundärzellen, (3) der Ableitungsdorn aus Messing im Inneren von AlkaliMangan-Zellen, (2) der Kohlestift der Zink-Kohle-Zellen. Der innere Aufbau von Ni-Cd- und Ni-MH-Zellen ist gleich. Um diese beiden Typen zu unterscheiden, wurden jeweils einigen Körnchen des Inhalts mit einem einfachen qualitativen Nachweis auf Cadmium getestet: Ein Filterpapierstreifen wird dazu mit stark ver- dünnter Salzsäure (ca. 0,5\%-ig) getränkt und einige Körnchen des Batterieinhalts darauf verteilt. Man lässt die Säure ca. eine Minute einwirken und hält dann den Streifen in eine Eprouvette, in der aus Schwefeleisen und verdünnter Salzsäure Schwefelwasserstoff entwickelt wird. Bei Anwesenheit von Cadmium färbt sich der Streifen in der Umgebung der Körnchen intensiv gelb (Ausfällung von Cadmiumsulfid).

Bei den Versuchen la und 1b wurden jeweils sämtliche $\mathrm{Ni}$-Cd-Batterien zu einer Analysenlösung verarbeitet. Die Zellen wurden - so weit möglich - zerlegt, d.h. die Elektroden aus den Bechern gezogen oder gerissen und entrollt. Alles abbröckelnde Material wurde gesammelt. Die Becher und Elektroden wurden mit konzentrierter Salzsäure so lange gespült bzw. darin getaucht, bis sie frei von Cd waren (Cd-Nachweis). Das beim Zerlegen angefallene Pulver wurde in der Spülsäure ca. eine halbe Stunde gekocht. Die Lösung wurde heiß über Schwarzbandfilter filtriert, der Filterkuchen gewaschen und verworfen.

Bei Versuch 2 wurden alle aus dem Schrott aussortierten Ni-Cd-Batterien nach dem Zufallsprinzip in zwei Gruppen aufgeteilt. Die beiden Gruppen wurden zur Gänze in Königswasser gelöst. Von diesen Lösungen (jeweils einige Liter) wurden Teilmengen entnommen und filtriert.

Die Bestimmung der Metallgehalte erfolgte wie folgt: Die Lösungen wurden im Verhältnis 1:100 verdünnt; anschließend wurde mittels ICP gegen säurematrixangepasste Standardlösungen der Metallgehalt gemessen.

\section{Ergebnisse}

\subsection{Batteriemengen und physischer Zustand der Batterien}

Lackierungen bzw. Folienüberzüge der aussortierten Batterien waren abgebrannt, die Oberflächen rostig und mit Schlackenresten verschmutzt. Bei An- lage 2 wurden kaum ganze Zn-C-Batterien gefunden, sondern oft nur noch angeschmolzene Bruchstücke oder die leeren Stahlblechmäntel. Billigfabrikate von $\mathrm{Zn}$-C-Zellen werden oft ohne Blechmantel erzeugt, sie sind mit Kunststofffolien überzogen. Diese Typen werden von der Magnetabscheidung nicht erfasst. Außerdem zerfallen diese Batterien, sobald der äußere Zinkbecher schmilzt. In der Schlacke findet man meist nur noch die Kohlestifte. Der Inhalt der Ni-Cd-Akkus war zu einem großen Teil pulverförmig. Die Ni-Aufsinterungen an den Elektroden waren meist intakt. Die Cd-Elektroden waren zum größten Teil in ein dunkelgraubraun, fast schwarzes Pulver sowie die gelochten Stahlfolien bzw. -gitter aufgelöst.

Tabelle 2 enthält die ermittelten Massenanteile von Batterien in den untersuchten Fraktionen. Die bemerkenswert kleinen Streuungen bei den Ni-Cd- und $\mathrm{Ni}-\mathrm{MH}$-Batteriegehalten für die Summe aus Grob- und Feinschrott bei Anlage 1 sind zufällig entstanden. Alleine auf Grund der geringen durchschnittlichen Stückzahlen ( 42 bei la und 46 bei $1 \mathrm{~b}$ ) wären mittlere relative Streuungen von mehr als $10 \%$ zu erwarten.

\subsection{Cadmiumgehalte der verbrannten $\mathrm{Ni}$-Cd-Batterien und der Schrotte}

In Tabelle 3 und 4 sind die Ergebnisse der chemischen Analysen zusammengefasst. Die deutlich höheren Werte von Anlage 2 sind möglicherweise auf die geringeren Verluste beim Öffnen der Batterien zurückzuführen.

\subsection{Beitrag der Ni-Cd-Batterein zum Cadmiumdurchsatz der Verbrennungsanlagen}

Aufgrund der unterschiedlichen Zielsetzung der Versuche 1 und 2 wurden nur bei Versuch 2 Massenbilanzen der Güter und ausgewählter Stoffe erstellt. Infolgedessen kann nur für diesen Versuch der Beitrag 
Tab. 2: Massenanteile der verschiedenen Batterien in elektromagnetisch aus MVA Schlacken abgeschiedenen Schrotten (Fe). Angaben in g/1.000kg FS

\begin{tabular}{|c|c|c|c|c|}
\hline Anlage 1 & AlkMn & $\mathrm{Zn}-\mathrm{C}$ & $\mathrm{Ni}-\mathrm{Cd}$ & $\mathrm{Ni}-\mathrm{MH}$ \\
\hline $\mathrm{Fe}>50 \mathrm{~mm} 1 \mathrm{a}$ & 120 & 0 & 125 & 0 \\
\hline $\mathrm{Fe}>50 \mathrm{~mm} 1 \mathrm{~b}$ & 350 & 14 & 144 & 0 \\
\hline Mittelw. \pm Stabw. & $240 \pm 160$ & $7 \pm 10$ & $134 \pm 14$ & - \\
\hline $\mathrm{Fe}<50 \mathrm{~mm} 1 \mathrm{a}$ & 10.600 & 1.730 & 2.350 & 1.170 \\
\hline $\mathrm{Fe}<50 \mathrm{~mm} 1 \mathrm{~b}$ & 8.980 & 1.350 & 2.850 & 1.480 \\
\hline Mittelw.土 Stabw. & $9.800 \pm 1.100$ & $1.540 \pm 270$ & $2.600 \pm 350$ & $1.320 \pm 210$ \\
\hline Fe gesamt 1a & 3.190 & 510 & 780 & 344 \\
\hline Fe gesamt $1 \mathrm{~b}$ & 2.340 & 320 & 770 & 340 \\
\hline Mittelw. \pm Stabw. & $2.770 \pm 600$ & $420 \pm 130$ & $770 \pm 3$ & $340 \pm 3$ \\
\hline \multicolumn{5}{|l|}{ Anlage 2} \\
\hline $\mathrm{Fe} 2 \mathrm{a}$ & 1.550 & 73 & 400 & 0 \\
\hline $\mathrm{Fe} 2 \mathrm{~b}$ & 1.350 & 0 & 620 & 230 \\
\hline Fe 2c & 1.070 & 120 & 1.270 & 0 \\
\hline $\mathrm{Fe} 2 \mathrm{~d}$ & 540 & 160 & 710 & 480 \\
\hline Mittelw. \pm Stabw. & $1.130 \pm 440$ & $90 \pm 70$ & $750 \pm 370$ & $180 \pm 230$ \\
\hline
\end{tabular}

Tab. 3: Cadmiumgehalte von Ni-Cd-Batterien aus Schrotten, die elektromagnetisch aus MVA Schlacken abgeschiedenen wurden. Angaben in g/100g FS

\begin{tabular}{|c|c|c|c|c|c|c|}
\hline \multicolumn{4}{|l|}{ Anlage 1} & \multicolumn{3}{|c|}{ Anlage 2} \\
\hline & $1 a$ & $1 b$ & Mittelw. \pm Stabw. & $2 a$ & $2 b$ & Mittelw. \pm Stabw. \\
\hline $\mathrm{Fe}>50 \mathrm{~mm}$ & 14,0 & -1 & - & 18,6 & 16,5 & $17,6 \pm 1,5$ \\
\hline $\mathrm{Fe}<50 \mathrm{~mm}$ & 10,5 & -1 & - & & & \\
\hline Mittelwert & 10,9 & 13,5 & $12,2 \pm 1,8$ & & & \\
\hline
\end{tabular}

Tab. 4: : Cadmiumgehalte von Schrotten, die elektromagnetisch aus MVA Schlacken abgeschiedenen wurden. Angaben in $\mathrm{mg} / \mathrm{kg}$ FS

Anlage 1

\begin{tabular}{lccc|c} 
& \multicolumn{3}{|c}{} & \multicolumn{2}{|c}{ Anlage 2 } \\
\hline $\mathrm{Fe}>\mathbf{5 0} \mathbf{~ m m}$ & 17 & 191 & $18 \pm 1$ & Mittelw. \pm Stabw. \\
\hline $\mathrm{Fe}<\mathbf{5 0} \mathbf{~ m m}$ & 250 & $380^{1}$ & $320 \pm 100$ & $130 \pm 30$ \\
\hline Mittelwert & 85 & 103 & $94 \pm 13$ & \\
\hline
\end{tabular}

${ }^{1} \mathrm{Ni}$-Cd-Batterien aus Schrottfraktion $>50 \mathrm{~mm}$ und $<50 \mathrm{~mm}$ gemeinsam aufgeschlossen und analysiert

der Ni-Cd-Akkus zum Gesamtgehalt von Cadmium im MVA-Input und die „batteriegebundene" Verteilung von Cadmium durch die Verbrennungsanlagen ermittelt werden.

In Anlage 2 trägt der aus der Schlacke abgeschiedene Schrott etwa $30 \%$ zur gesamten Outputfracht an Cadmium bei. Dies überrascht, da der Schrott nur 3,5\% der Masse des verbrannten Mülls darstellt. Der Grund dafür sind die im Schrott befindlichen Batterien und deren Reste.
Diese Batterien sind mit rund $5 \mathrm{mg} / \mathrm{kg}$ für ein Drittel des Cadmiumgehaltes des verbrannten Mülls von $15 \mathrm{mg} / \mathrm{kg}$ verantwortlich. Batterien sind im Schrott um etwa Faktor 9 gegenüber dem Müllinput angereichert. In der entschrotteten Schlacke wurden weitere $\mathrm{Ni}$-Cd-Akkus aufgefunden, die durch die Magnetabscheidung nicht aussortiert wurden. Diese tragen zusätzliche $1,5 \mathrm{mg} / \mathrm{kg}$ zum Cadmiumgesamtgehalt des Müllinputs bei. Insgesamt können demnach ca. 6,5 mg/kg, d. h. rund $40 \%$, vom Cadmiumgehalt des Mülls den Ni-Cd-Batterien zugeordnet werden. Diese Zahl entspricht einem Minimum, da unbekannt ist, wieviel Cadmium während der Verbrennung aus den Batterien austritt.

Bei der Schlackenaufbereitung auf Anlage 1 wurden aus der Schlacke nur 5,5\% als Fe-Schrott abgeschieden (Anlage 2: 11,9\%). Hochgerechnet mit der Annahme, dass $30 \%$ des verbrannten Mülls über den Rost ausgetragen werden (zum Vergleich Anlage 2: 29,6\%), beträgt die Masse des Schrottoutput nur 1,6\% bezogen auf den Müllinput. Für den Beitrag der Ni-Cd-Batterien im FeSchrott zum Gesamtgehalt an Cadmium im Müllinput ergibt sich daraus ein Wert von $1,5 \mathrm{mg} / \mathrm{kg}$.

\section{Schlussfolgerungen}

Das Resultat dieser Arbeit zeigt, dass Cd in Schlacken und daraus abgetrennten Schrotten ein relevantes Thema ist: Insbesondere Ni-Cd-Akkumulatoren verursachen hohe Cadmiumgehalte in elektromagnetisch aus der Schlacke abgeschiedenen Schrotten der Müllverbrennung. Die durchschnittlichen Cadmiumgehalte in Schrotten von MVAen liegen im Bereich um $100 \mathrm{mg} / \mathrm{kg}$. Im Jahr 2005 wurden ca. 1,1 Mio. Tonnen Abfälle in Rostfeuerungen verbrannt (Böhmer et al., 2007). Bei einem Schrottanfall von 2,5\% der Inputmasse wären etwa 3 Tonnen Cadmium in den Schrotthandel gelangt. Derzeit ist nicht bekannt, wo die mit dem Schrott aus der Müllverbrennung ausgebrachte Menge an Cadmium verbleibt. Es ist zu prüfen, ob Cadmium beim Shredderprozess in den Shreddersand übergeht, oder ob es in der Eisenfraktion verbleibt und in die Stahlproduktion gelangt.

Es ist unbekannt, wie viel des ursprünglich in den Batterien enthaltenen Cadmiums im Feuerraum aus den Zellen ausgetrieben wird, bzw. wie groß der Massenverlust der Zellen insgesamt während der Verbrennung ist. Die Ergebnisse von Versuch 2 - sowohl die Cadmiumgehalte der analysierten Batterien als auch die Beiträge der Batterien zum Gasamtgehalt des verbrannten Abfalls - sprechen für einen geringen Verlust. Auch Bunge et al. (2008) meinen, dass „der Großteil des Cadmiums in den Batterien verbleibt und entweder mit der Schlacke deponiert oder mit dem Stahlschrott exportiert würde“. Die quantitativen Angaben dieser Studie zur Rückgewinnung 
von Batterien aus KVA-Schlacken sind noch ausständig.

Die vorliegende Arbeit zeigt, dass nur mit einem relativ großen Aufwand bezüglich Probenahme und Probenaufbereitung zuverlässige Cadmiumwerte von aus Müllschlacken abgeschiedenen Schrotten gewonnen werden können. Die richtige Bestimmung des Massenanteils und der Beiträge zum Cadmiumgehalt von Schlacke und Schrott bedarf relativ vieler Handarbeit, eine Tätigkeit, die sich im routinemäßigen analytischen Betrieb meist als zu teuer erweist. Auf Grund der hohen Konzentration von Cadmium in den Batterien im Vergleich zu den übrigen Gemengeteilen, reichen einige wenige Stück pro Tonne (bei Schlacken) bzw. einige zig Stück pro Tonne (be Schrotten) um relevante Beiträge zur CdFracht des Gutes bzw. zum Cd-Umsatz einer Müllverbrennungsanlage zu leis- ten. Nach einer Faustregel sind, um den Probenahmefehler mit $90 \%$ Wahrscheinlichkeit unter $15 \%$ zu halten, Probenmassen erforderlich, die es erlauben, im Schnitt etwa 120 der analytreichen Stücke in einer Probe anzutreffen. Für die genaue Bestimmung sind daher allein auf Grund dieser in der Konstitution des Ma-

terials begründeten Heterogenität Probenmassen von einigen Tonnen be Schrotten und zig Tonnen bei Schlacken notwendig.

Korrespondenz:

Technische Universität Wien

Institut für Wassergüte, Ressourcenmanagement und Abfallwirtschaft

\section{LITERATUR}

Böhmer S, Kügler I, Stoiber H, Walter B 2007) Ab Kugrerennung in Osterreich Statusbericht 2006. Report REP-0113, Umweltbundesamt $\mathrm{GmbH}$ Wien. Brunner PH, Zobrist J (1983) Die Müllverbrennung als Quelle von Metallen in der Umwelt, Müll und Abfall, 15(9), 221-226. Bunge R, Krebs A, Meyer MK (2008) Unkonventionelles Recycling-Batterierückgewinnung aus KVA-Schlacke als "Schadensbegrenzung Maufmars (1997) Cadmium - Stofffluss, Ziegler M (1997) Cadmelt Nr. 295. Bundanalye. SchriftenWald und Landschaft (BUWAL), Bern.
Skutan S, Brunner PH (2009) Material Balance of mechanical-biological MSW treatment plants, Manuskript in Ausarbeitung.

Vanzetta GM (2002) Contributo alla caratterizzazione, separazione meccanica e recupero di metalli dai fifiuti urbani. il caso di alluminio e cadmio (Beitrag zur Bestimmung, mechanischen Abtrennung und Rückgewinnung von Metallen aus Restmüll am Beispiel von Aluminium und Cadmium), Diplomarbeit ausgeführt am Institu Abfallwirtschaft, TU Wien. 